\title{
Existence of positive solutions of discrete third-order three-point BVP with sign-changing Green's function
}

\author{
Youji Xu ${ }^{1 *}$ (D), Weiwei Tian' and Chenghua Gao'
}

\section{"Correspondence:}

xuyj@nwnu.edu.cn

${ }^{1}$ College of Mathematics and

Statistics, Northwest Normal

University, Lanzhou, P.R. China

\section{Abstract}

Consider positive solutions and multiple positive solutions for a discrete nonlinear third-order boundary value problem

$$
\left\{\begin{array}{l}
\Delta^{3} u(t-1)=a(t) f(t, u(t)), \quad t \in[1, T-2]_{\mathbb{Z}}, \\
\Delta u(0)=u(T)=0, \quad \Delta^{2} u(\eta)-\alpha \Delta u(T-1)=0,
\end{array}\right.
$$

which has the sign-changing Green's function. Here $T>8$ is a positive integer, $[1, T-1]_{\mathbb{Z}}=\{1,2, \ldots, T-2\}, \alpha \in\left[0, \frac{1}{T-1}\right), a:[0, T-2]_{\mathbb{Z}} \rightarrow(0,+\infty)$, $f:[1, T-2]_{\mathbb{Z}} \times[0, \infty) \rightarrow[0, \infty)$ is continuous.

Keywords: Discrete third-order three-point boundary value problem; Positive solutions; Cone; Fixed point; Sign-changing Green's function

\section{Introduction}

Multi-point boundary value problems for differential equations have a wide application in computational physics, economics, and modern biological fields [1]. In 1992, Gupta [2] studied solvability of differential equation three-point boundary value problem. Soon afterwards, there arose many results on multi-point nonlinear boundary value problems [3-6]. In 1999, Ma [7] studied the existence of positive solution for a second-order differential equation three-point boundary value problem. Thereafter, many results for the existence of positive solutions on multi-point boundary value problems have been studied [7-19]. With the development of the computing science and the computer simulation, multi-point boundary value problems should be discretized, so we need to study corresponding difference equation [20-26]. In 1998, by using Krasnosel'skii's fixed point theorem, Agarwal and Henderson [24] studied the discrete problem

$$
\left\{\begin{array}{l}
\Delta^{3} u(t-1)=\lambda a(t) f(t, u(t)), \quad t \in[2, T]_{\mathbb{Z}}, \\
u(0)=u(1)=u(T+3)=0 .
\end{array}\right.
$$

They obtained the existence of positive solutions in two cases for $\lambda=1$ and $\lambda \neq 1$. Later, there were many interesting results on the positive solutions to the discrete boundary

(c) The Author(s) 2019. This article is distributed under the terms of the Creative Commons Attribution 4.0 International License (http://creativecommons.org/licenses/by/4.0/), which permits unrestricted use, distribution, and reproduction in any medium, provided you give appropriate credit to the original author(s) and the source, provide a link to the Creative Commons license, and indicate if changes were made. 
value problems, see, for instance, [23-26] and the references therein. It is noted that Green's functions are positive in most of these results. However, when the Green's function is sign-changing, could we also obtain the existence of positive solutions to these kinds of problems?

In 2015, by using the Guo-Krasnosel'skii fixed point theorem, Wang and Gao [25] studied the existence of positive solutions to the discrete third-order three-point boundary value problem

$$
\left\{\begin{array}{l}
\Delta^{3} u(t-1)+a(t) f(t, u(t))=0, \quad t \in[1, T-1]_{\mathbb{Z}} \\
u(0)=\Delta u(T)=\Delta^{2} u(\eta)=0
\end{array}\right.
$$

where $\eta \in\left[1,\left[\frac{3 T^{2}-3 T-2}{6 T+3}\right]\right]_{\mathbb{Z}}$ and the Green's function is sign-changing. In 2016, Geng and Gao [26], by using the Guo-Krasnosel'skii fixed point theorem, studied the discrete thirdorder three-point boundary value problem

$$
\left\{\begin{array}{l}
\Delta^{3} u(t-1)+\lambda a(t) f(t, u(t))=0, \quad t \in[1, T-1]_{\mathbb{Z}}, \\
u(0)=\Delta u(t)=\Delta^{2} u(\eta)=0,
\end{array}\right.
$$

when $f$ satisfies some superlinear and sublinear condition on 0 and $\infty$. For the continuous case, which has the sign-changing Green's function, one can see [27-29] and the references therein. Inspired by the above works, in this paper, we consider the existence and multiple positive solutions to the following discrete nonlinear third-order three-point BVP:

$$
\left\{\begin{array}{l}
\Delta^{3} u(t-1)=a(t) f(t, u(t)), \quad t \in[1, T-2]_{\mathbb{Z}}, \\
\Delta u(0)=u(T)=0, \quad \Delta^{2} u(\eta)-\alpha \Delta u(T-1)=0,
\end{array}\right.
$$

where $T>8$ is a positive integer, $\alpha \in\left[0, \frac{1}{T-1}\right), a:[1, T-2]_{\mathbb{Z}} \rightarrow(0,+\infty), f:[1, T-2]_{\mathbb{Z}} \times$ $[0, \infty) \rightarrow[0, \infty)$ is continuous, $\eta$ satisfies

$\left(H_{0}\right) \eta \in\left[\left\lfloor\frac{T-2}{2}\right\rfloor+1, T-2\right]_{\mathbb{Z}}$.

Under assumption $\left(H_{0}\right)$, the Green's function of (1.1) changes its sign. The proof of our main results is based upon the following well-known Guo-Krasnoselskii fixed point theorems [30].

Theorem 1.1 Let E be a Banach space and $K \subset E$ be a cone. Assume that $\Omega_{1}, \Omega_{2}$ are open bounded subsets of $E$ with $0 \in \Omega_{1}, \bar{\Omega}_{1} \subset \Omega_{2}$. If

$$
A: K \cap\left(\bar{\Omega}_{2} \backslash \Omega_{1}\right) \rightarrow K
$$

is a completely continuous operator such that

(i) $\|A u\| \leq\|u\|, u \in K \cap \partial \bar{\Omega}_{1}$, and $\|A u\| \geq\|u\|, u \in K \cap \partial \bar{\Omega}_{2}$, or

(ii) $\|A u\| \geq\|u\|, u \in K \cap \partial \bar{\Omega}_{1}$, and $\|A u\| \leq\|u\|, u \in K \cap \partial \bar{\Omega}_{2}$, then $A$ has a fixed point in $K \cap\left(\bar{\Omega}_{2} \backslash \Omega_{1}\right)$.

Theorem 1.2 Let E be a Banach space and $K$ be a cone in E. For some $p>0$, define $K_{p}=\{x \in$ $K \mid\|x\| \leq p\}$. Assume that $A: K_{p} \rightarrow K$ is a compact operator; if $x \in \partial K_{p}=\{x \in k \mid\|x\|=p\}$, $A x \neq x$, we have 
(i) For $\forall x \in \partial K_{p}$, if $\|A x\| \geq\|x\|$, then $i\left(A, K_{p}, K\right)=0$,

(ii) For $\forall x \in \partial K_{p}$, if $\|A x\| \leq\|x\|$, then $i\left(A, K_{p}, K\right)=1$.

\section{Preliminaries}

Lemma 2.1 Suppose that $\left(H_{0}\right)$ holds. Then the linear problem

$$
\left\{\begin{array}{l}
\Delta^{3} u(t-1)=y(t), \quad t \in[1, T-2]_{\mathbb{Z}} \\
\Delta u(0)=u(T)=0, \quad \Delta^{2} u(\eta)-\alpha \Delta u(T-1)=0
\end{array}\right.
$$

has a unique solution

$$
u(t)=\sum_{s=1}^{T-2} G(t, s) y(s)
$$

where

$$
\begin{aligned}
& G(t, s)=g(t, s)+k(t, s)+ \begin{cases}\frac{T(T-1)-t(t-1)}{2-2 \alpha(T-1)}, & s \leq \eta, \\
0, & \eta<s,\end{cases} \\
& g(t, s)=-\frac{\alpha(T-s-1)[T(T-1)-t(t-1)]}{2-2 \alpha(T-1)}-\frac{(T-s)(T-s-1)}{2}, \\
& k(t, s)= \begin{cases}\frac{(t-s)(t-s-1)}{2}, & 0<s \leq t-2 \leq T-2, \\
0, & 0 \leq t-2<s \leq T-2,\end{cases}
\end{aligned}
$$

and

$$
G(0, s)=G(1, s)= \begin{cases}\frac{-\alpha T(T-1)(T-s-1)+T(T-1)}{2-2 \alpha(T-1)}-\frac{(T-1)(T-s-1)}{2}, & s \leq \eta, \\ \frac{-\alpha T(T-1)(T-s-1)}{2-2 \alpha(T-1)}-\frac{(T-1)(T-s-1)}{2}, & \eta<s .\end{cases}
$$

Proof Summing both sides of equation (2.1) from $s=1$ to $s=t-1$, we get

$$
\Delta^{2} u(t-1)=\Delta^{2} u(0)+\sum_{s=1}^{t-1} y(s)
$$

and

$$
\Delta u(t-1)=(t-1) \Delta^{2} u(0)+\sum_{s=1}^{t-2}(t-s-1) y(s) .
$$

Then summing both sides from $\tau=1$ to $\tau=t$, we get

$$
u(t)=u(0)+\frac{t(t-1)}{2} \Delta^{2} u(0)+\sum_{s=1}^{t-2} \frac{(t-s)(t-s-1)}{2} y(s) .
$$


From boundary conditions $\Delta u(0)=u(T)=0, \Delta^{2} u(\eta)-\alpha \Delta u(T-1)=0$, we have

$$
\left\{\begin{array}{l}
u(0)+\frac{T(T-1)}{2} \Delta^{2} u(0)+\sum_{s=1}^{T-2} \frac{(T-s)(T-s-1)}{2} y(s)=0 \\
\Delta^{2} u(0)+\sum_{s=1}^{\eta} y(s)-\alpha(T-1) \Delta^{2} u(0)-\alpha \sum_{s=1}^{T-2}(T-s-1) y(s)=0 .
\end{array}\right.
$$

Furthermore, we get

$$
\left\{\begin{aligned}
u(0)= & \sum_{s=1}^{T-2} \frac{-\alpha T(T-1)(T-s-1)}{2-2 \alpha(T-1)} y(s)+\sum_{s=1}^{\eta} \frac{T(T-1)}{2-2 \alpha(T-1)} y(s) \\
& -\sum_{s=1}^{T-2} \frac{(T-s)(T-s-1)}{2} y(s) \\
\Delta^{2} u(0)= & \sum_{s=1}^{T-2} \frac{\alpha(T-s-1)}{1-\alpha(T-1)} y(s)-\sum_{s=1}^{\eta} \frac{1}{1-\alpha(T-1)} y(s) .
\end{aligned}\right.
$$

Then we have

$$
\begin{aligned}
u(t)= & \sum_{s=1}^{T-2}\left(-\frac{\alpha(T-s-1)[T(T-1)-t(t-1)]}{2-2 \alpha(T-1)}-\frac{(T-s)(T-s-1)}{2}\right) y(s) \\
& +\sum_{s=1}^{t-2} \frac{(t-s)(t-s-1)}{2} y(s) \\
& +\sum_{s=1}^{\eta} \frac{T(T-1)-t(t-1)}{2-2 \alpha(T-1)} y(s) .
\end{aligned}
$$

Lemma 2.2 Suppose that $\left(H_{0}\right)$ holds. Then the Green's function $G(t, s)$ changes its sign on $[0, T]_{\mathbb{Z}} \times[0, T]_{\mathbb{Z}}$. More precisely,

(i) If $s \in[1, \eta]_{\mathbb{Z}}$, then $\Delta_{t} G(t, s) \leq 0, G(t, s) \geq 0$ for $\forall t \in[0, T]_{\mathbb{Z}}$. Furthermore,

$$
\begin{aligned}
& \min _{t \in[0, T]_{\mathbb{Z}}} G(t, s)=G(T, s)=0, \\
& \max _{t \in[0, T]_{\mathbb{Z}}} G(t, s)=G(0, s) \leq \frac{T(T-1)(1+\alpha \eta)}{1-\alpha(T-1)} .
\end{aligned}
$$

(ii) If $s \in[\eta+1, T-2]_{\mathbb{Z}}$, then $\Delta_{t} G(t, s) \geq 0, G(t, s) \leq 0$ for $\forall t \in[0, T]_{\mathbb{Z}}$. Furthermore,

$$
\begin{aligned}
& \max _{t \in[0, T]_{\mathbb{Z}}} G(t, s)=G(T, s)=0, \\
& \min _{t \in[0, T]_{\mathbb{Z}}} G(t, s)=G(0, s) \geq \frac{-T(T-1)(1+\alpha \eta)}{1-\alpha(T-1)} .
\end{aligned}
$$

Proof (i) If $s \in[1, \eta]_{\mathbb{Z}}$, we have

$$
\Delta_{t} G(t, s)= \begin{cases}\frac{\alpha t(T-s-1)-2 t}{1-\alpha(T-1)}, & t-2 \leq s \\ \frac{\alpha t(T-s-1)-2 t}{1-\alpha(T-1)}+t-s, & s \leq t-2\end{cases}
$$

If $s \leq t-2$, since $\alpha(T-1)-1<0$, we get

$$
\begin{aligned}
\Delta_{t} G(t, s) & =\frac{2 \alpha t(T-s-1)-2 t}{2-2 \alpha(T-1)}+t-s \\
& =\frac{2 \alpha t(T-s-1)-2 t+(t-s)(2-2 \alpha(T-1))}{2-2 \alpha(T-1)}
\end{aligned}
$$




$$
\begin{aligned}
& =\frac{-2 s \alpha+2 s[\alpha(T-1)-1]}{2-2 \alpha(T-1)} \\
& <0
\end{aligned}
$$

If $t-2 \leq s$, since $\alpha<\frac{1}{T-1}$, so $2-2 \alpha(T-1)>0, \alpha(T-s-1)-1<0$, we have

$$
\Delta_{t} G(t, s)=\frac{2 \alpha t(T-s-1)-2 t}{2-2 \alpha(T-1)}<0 .
$$

For $\forall t \in[0, T-1]_{\mathbb{Z}}, \Delta_{t} G(t, s) \leq 0$. If $s \in[1, \eta]_{\mathbb{Z}}, G(t, s) \geq 0$. So

$$
\begin{aligned}
\min _{t \in[0, T]_{\mathbb{Z}}} G(t, s) & =G(T, s)=0, \\
\max _{t \in[0, T]_{\mathbb{Z}}} G(t, s) & =G(0, s) \\
& =\frac{T(T-1)[1-\alpha(T-s-1)]}{2-2 \alpha(T-1)}-\frac{(T-s)(T-s-1)}{2} \\
& \leq \frac{T(T-1)[1-\alpha(T-\eta-1)]}{2-2 \alpha(T-1)}-\frac{(T-\eta)(T-\eta-1)}{2} \\
& \leq \frac{T(T-1)(1+\alpha \eta)}{1-\alpha(T-1)} .
\end{aligned}
$$

(ii) If $s \in[\eta+1, T]_{\mathbb{Z}}$, we have

$$
\Delta_{t} G(t, s)= \begin{cases}\frac{2 \alpha t(T-s-1)}{2-2 \alpha(T-1)}, & s \geq t-2, \\ \frac{2 \alpha t(T-s-1)}{2-2 \alpha(T-1)}+t-s, & s \leq t-2 .\end{cases}
$$

If $t-2 \leq s$, since $\alpha<\frac{1}{T-1}, 2-2 \alpha(T-1)>0$, we have

$$
\Delta_{t} G(t, s)=\frac{2 \alpha t(T-s-1)}{2-2 \alpha(T-1)}>0 .
$$

If $s \leq t-2$, then $t-s>0$, so

$$
\Delta_{t} G(t, s)=\frac{2 \alpha t(T-s-1)}{2-2 \alpha(T-1)}+t-s>0 .
$$

If $s \in[\eta+1, T]_{\mathbb{Z}}$, for $\forall t \in[0, T]_{\mathbb{Z}}$, we have

$$
\begin{aligned}
\max _{t \in[0, T]_{\mathbb{Z}}} G(t, s) & =G(T, s)=0 \\
\min _{t \in[0, T]_{\mathbb{Z}}} G(t, s) & =G(0, s) \\
& =\frac{-\alpha T(T-1)(T-s-1)}{2-2 \alpha(T-1)}-\frac{(T-s)(T-s-1)}{2} \\
& \geq \frac{-\alpha T(T-1)(T-\eta-1)}{2-2 \alpha(T-1)}-\frac{(T-\eta)(T-\eta-1)}{2} \\
& \geq \frac{-T(T-1)(1+\alpha \eta)}{1-\alpha(T-1)} .
\end{aligned}
$$


In conclusion, if $s \in[1, \eta]_{\mathbb{Z}}, G(t, s)$ is decreasing, then $G(t, s) \geq 0$, since $\min _{t \in[0, T]_{\mathbb{Z}}} G(t, s)=$ 0 ; if $s \in[\eta+1, T]_{\mathbb{Z}}, G(t, s)$ is increasing, then $G(t, s) \leq 0$ since $\max _{t \in[0, T]_{\mathbb{Z}}} G(t, s)=0$. So, the Green's function $G(t, s)$ is a sign-changing function on $[0, T]_{\mathbb{Z}} \times[0, T]_{\mathbb{Z}}$.

Remark Now, we give a brief explanation for the reason why we choose

$$
\eta \in\left[\left\lfloor\frac{T-2}{2}\right\rfloor+1, T-2\right]_{\mathbb{Z}}
$$

Consider the problem

$$
\left\{\begin{array}{l}
\Delta^{3} u(t-1)=1, \quad t \in[1, T-2]_{\mathbb{Z}}, \\
\Delta u(0)=u(T)=0, \quad \Delta^{2} u(\eta)-\alpha \Delta u(T-1)=0 .
\end{array}\right.
$$

From Lemma 2.1, we get

$$
\begin{aligned}
u(t)= & \frac{1}{12-12 \alpha(T-1)}\{3 \alpha[t(t-1)-T(T-1)](T-1)(T-2) \\
& +6 \eta[T(T-1)-t(t-1)]-2 T(T-1)(T-2)[1-\alpha(T-1)] \\
& +2 t(t-1)(t-2)[1-\alpha(T-1)]\} \\
= & \frac{\phi(t)}{12-12 \alpha(T-1)}
\end{aligned}
$$

where

$$
\begin{aligned}
\phi(t)= & 3 \alpha[t(t-1)-T(T-1)](T-1)(T-2)+6 \eta[T(T-1)-t(t-1)] \\
& -2 T(T-1)(T-2)[1-\alpha(T-1)]+2 t(t-1)(t-2)[1-\alpha(T-1)] .
\end{aligned}
$$

Clearly, $u(t) \geq 0$ is equivalent to $\phi(t) \geq 0$, and

$$
\Delta \phi(t)=6 t\{(t-1)[1-\alpha(T-1)]-2 \eta+\alpha(T-1)(T-2)\} .
$$

Let $\Delta \phi(t)=0$. Then $t=0$ or $t=1+\frac{2 \eta-\alpha(T-1)(T-2)}{1-\alpha(T-1)}$. Therefore,

$$
\Delta \phi(t)>0 \quad \Leftrightarrow \quad t>\frac{2 \eta+1-\alpha(T-1)^{2}}{1-\alpha(T-1)} .
$$

Now, we claim that if (2.3) holds, then $\phi(t)$ is a positive solution of (2.4). In fact, if (2.3) holds, then $\frac{2 \eta+1-\alpha(T-1)^{2}}{1-\alpha(T-1)} \geq T-1$ in this case, this implies that

$$
\Delta \phi(t) \leq 0, \quad t \in[0, T-1]_{\mathbb{Z}}
$$

More precisely, $\Delta \phi(0)=0, \Delta \phi(t)<0$ for $t \in[1, T-2]_{\mathbb{Z}}$ and $\Delta \phi(T-1)<0$ for $\frac{2 \eta+1-\alpha(T-1)^{2}}{1-\alpha(T-1)}>$ $T-1$ and $\Delta \phi(T-1)=0$ for $\frac{2 \eta+1-\alpha(T-1)^{2}}{1-\alpha(T-1)}=T-1$. Therefore, $\phi(t)$ is a positive solution of the linear problem (2.4) since $\phi(T)=0$. 
Let

$$
E=\left\{u:[0, T]_{\mathbb{Z}} \rightarrow \mathbb{R} \mid \Delta u(0)=u(T), \Delta^{2} u(\eta)-\alpha \Delta u(T-1)=0\right\} .
$$

Then $E$ is a Banach space with norm $\|u\|=\max _{t \in[0, T]_{\mathbb{Z}}}|u(t)|$.

Let

$$
K_{0}=\left\{y \in E: y(t) \geq 0, t \in[0, T]_{\mathbb{Z}} ; \Delta y(t) \leq 0, t \in[0, T-1]_{\mathbb{Z}}\right\} .
$$

Then $K_{0}$ is a cone in $E$.

Lemma 2.3 Let $\left(H_{0}\right)$ hold. If $y \in K_{0}$, then the solution $u(t)$ of problem (2.1) belongs to $K_{0}$, i.e., $u \in K_{0}$. Furthermore, $u(t)$ is concave on $[0, \eta]_{\mathbb{Z}}$.

Proof Firstly, if $0 \leq t-2 \leq \eta$, then

$$
\begin{aligned}
u(t)= & \sum_{s=\eta+1}^{T-2}\left\{\frac{-\alpha(T-s-1)[T(T-1)-t(t-1)]}{2-2 \alpha(T-1)}-\frac{(T-s)(T-s-1)}{2}\right\} y(s) \\
& +\sum_{s=1}^{\eta}\left\{\frac{[1-\alpha(T-s-1)][T(T-1)-t(t-1)]}{2-2 \alpha(T-1)}-\frac{(T-s)(T-s-1)}{2}\right\} y(s) \\
& +\sum_{s=1}^{t-2} \frac{(t-s)(t-s-1)}{2} y(s) .
\end{aligned}
$$

Then we obtain that

$$
\begin{aligned}
\Delta u(t)= & u(t+1)-u(t) \\
= & -\sum_{s=1}^{\eta} \frac{t[1-\alpha(T-s-1)]}{1-\alpha(T-1)} y(s)+\sum_{s=1}^{t-2}(t-s) y(s)+y(t-1)+\sum_{s=\eta+1}^{T-2} \frac{\alpha t(T-s-1)}{1-\alpha(T-1)} y(s) \\
= & \sum_{s=1}^{t-2} \frac{-\alpha t s+s(\alpha(T-1)-1)}{1-\alpha(T-1)} y(s)-\sum_{s=t-1}^{\eta} \frac{t[1-\alpha(T-s-1)]}{1-\alpha(T-1)} y(s)+y(t-1) \\
& +\sum_{s=\eta+1}^{T-2} \frac{\alpha t(T-s-1)}{1-\alpha(T-1)} y(s) .
\end{aligned}
$$

Since $y \in K_{0}$, we know that $y$ is decreasing on $[0, T]_{\mathbb{Z}}$. That is to say, $y(t) \geq y(\eta)$ for $t \leq \eta$ and $y(t) \leq y(\eta)$ for $t \geq \eta$. Therefore, if $t-1 \leq \eta$, then

$$
\begin{aligned}
& \Delta u(t) \\
& =\sum_{s=1}^{t-1} \frac{-\alpha t s+s(\alpha(T-1)-1)}{1-\alpha(T-1)} y(s)-\sum_{s=t-1}^{\eta} \frac{t[1-\alpha(T-s-1)]}{1-\alpha(T-1)} y(s) \\
& \quad+\sum_{s=\eta+1}^{T-2} \frac{\alpha t(T-s-1)}{1-\alpha(T-1)} y(s)
\end{aligned}
$$




$$
\begin{aligned}
& \leq y(\eta)\left\{\sum_{s=1}^{t-1} \frac{-\alpha t s+s(\alpha(T-1)-1)}{1-\alpha(T-1)}-\sum_{s=t-1}^{\eta} \frac{t[1-\alpha(T-s-1)]}{1-\alpha(T-1)}+\sum_{s=\eta+1}^{T-2} \frac{\alpha t(T-s-1)}{1-\alpha(T-1)}\right\} \\
& =y(\eta)\left\{-\sum_{s=1}^{\eta} \frac{t[1-\alpha(T-s-1)]}{1-\alpha(T-1)}+\sum_{s=1}^{t-1}(t-s)+\sum_{s=\eta+1}^{T-2} \frac{\alpha t(T-s-1)}{1-\alpha(T-1)}\right\} .
\end{aligned}
$$

If $t-1>\eta$, then $y(t-1) \leq y(\eta)$. Therefore, by (2.5), no matter $t-1 \leq \eta$ or $t-1>\eta$, we always have

$$
\begin{aligned}
\Delta u(t) & \leq y(\eta)\left\{-\sum_{s=1}^{\eta} \frac{t[1-\alpha(T-s-1)]}{1-\alpha(T-1)}+\sum_{s=1}^{t-1}(t-s)+\sum_{s=\eta+1}^{T-2} \frac{\alpha t(T-s-1)}{1-\alpha(T-1)}\right\} \\
& =\frac{t y(\eta)}{1-\alpha(T-1)}\left\{-\eta+\frac{(t-1)[1-\alpha(T-1)]}{2}+\frac{\alpha(T-1)(T-2)}{2}\right\} \\
& =\frac{1}{1-\alpha(T-1)}[-2 \eta+(t-1)+\alpha(T-1)(T-t-1)] .
\end{aligned}
$$

Combining this with $\eta \geq \frac{T-2}{2}$, we have

$$
\Delta u(t) \leq(T-t-1)(\alpha(T-1)-1)<0 .
$$

Furthermore, we have

$$
\begin{array}{rl}
\Delta^{2} & u(t-1) \\
& =-\sum_{s=1}^{\eta} \frac{1-\alpha(T-s-1)}{1-\alpha(T-1)} y(s)+\sum_{s=1}^{t} y(s)+\sum_{s=\eta+1}^{T-2} \frac{\alpha(T-s-1)}{1-\alpha(T-1)} y(s) \\
& =-\sum_{s=1}^{\eta} \frac{1-\alpha(T-s-1)}{1-\alpha(T-1)} y(s)+\sum_{s=1}^{t-2} y(s)+y(t-1)+y(t)+\sum_{s=\eta+1}^{T-2} \frac{\alpha(T-s-1)}{1-\alpha(T-1)} y(s) .
\end{array}
$$

Now, if $t-1 \leq \eta$ and $t \leq \eta$, then

$$
\begin{aligned}
& \Delta^{2} u(t-1) \\
& \quad \leq-\sum_{s=1}^{t-2} \frac{s \alpha}{1-\alpha(T-1)} y(s)-\sum_{s=t-1}^{\eta} \frac{1-\alpha(T-s-1)}{1-\alpha(T-1)} y(s)+\sum_{s=\eta+1}^{T-2} \frac{\alpha(T-s-1)}{1-\alpha(T-1)} y(s)+2 y(\eta) .
\end{aligned}
$$

Combining this with the monotonicity of $y$, we get that

$$
\begin{aligned}
& \Delta^{2} u(t-1) \\
& \quad \leq y(\eta)\left\{-\sum_{s=1}^{t-2} \frac{s \alpha}{1-\alpha(T-1)}-\sum_{s=t-1}^{\eta} \frac{1-\alpha(T-s-1)}{1-\alpha(T-1)}+\sum_{s=\eta+1}^{T-2} \frac{\alpha(T-s-1)}{1-\alpha(T-1)}+2\right\} \\
& \quad=y(\eta)\left\{-\sum_{s=1}^{\eta} \frac{1-\alpha(T-s-1)}{1-\alpha(T-1)}+t+\sum_{s=\eta+1}^{T-2} \frac{\alpha(T-s-1)}{1-\alpha(T-1)}\right\} \\
& =\frac{y(\eta)}{1-\alpha(T-1)}\left\{-\eta+\frac{t[1-\alpha(T-1)]}{2}+\frac{\alpha(T-1)}{2}\right\}
\end{aligned}
$$




$$
\begin{aligned}
& =\frac{y(\eta)}{1-\alpha(T-1)} \frac{-2 \eta+2 t+\alpha(T-1)(T-2)}{2} \\
& \leq 0
\end{aligned}
$$

For other cases, $t-1 \leq \eta<t$ and $t-1>\eta$, we could also obtain $\Delta^{2} u(t-1) \leq 0$ for $0 \leq$ $t-2 \leq \eta$.

Secondly, if $\eta<t-2 \leq T-2$, then

$$
\begin{aligned}
u(t)= & \sum_{s=t-1}^{T-2}\left\{-\frac{\alpha(T-s-1)[T(T-1)-t(t-1)]}{2-2 \alpha(T-1)}-\frac{(T-s)(T-s-1)}{2}\right\} y(s) \\
& +\sum_{s=1}^{t-2}\left\{\frac{-\alpha(T-s-1)[T(T-1)-t(t-1)]}{2-2 \alpha(T-1)}-\frac{(T-s)(T-s-1)}{2}\right. \\
& \left.+\frac{(t-s)(t-s-1)}{2}\right\} y(s)+\sum_{s=1}^{\eta} \frac{T(T-1)-t(t-1)}{2-2 \alpha(T-1)} y(s)
\end{aligned}
$$

and

$$
\begin{aligned}
\Delta u(t) & =\sum_{s=1}^{T-2} \frac{\alpha t(T-s-1)}{1-\alpha(T-1)} y(s)+\sum_{s=1}^{t-1}(t-s) y(s)-\sum_{s=1}^{\eta} \frac{t}{1-\alpha(T-1)} y(s) \\
& =-\sum_{s=1}^{\eta} \frac{\alpha t s+s(1-\alpha(T-1))}{1-\alpha(T-1)} y(s)+\sum_{s=\eta+1}^{T-2} \frac{\alpha t(T-s-1)}{(1-\alpha(T-1))} y(s)+\sum_{s=\eta+1}^{t-1}(t-s) y(s) .
\end{aligned}
$$

Combining this with the fact that $y \in K_{0}$ and the monotonicity of $y$, we get that

$$
\begin{aligned}
\Delta u(t) & \leq y(\eta)\left\{-\sum_{s=1}^{\eta} \frac{\alpha t s+s(1-\alpha(T-1))}{1-\alpha(T-1)}+\sum_{s=\eta+1}^{T-2} \frac{\alpha t(T-s-1)}{1-\alpha(T-1)}+\sum_{s=\eta+1}^{t-1}(t-s)\right\} \\
& =\frac{y(\eta)}{2-2 \alpha(T-1)}[\alpha t(T-1)(T-2)+t(t-1)(1-\alpha(T-1))-2 t \eta] .
\end{aligned}
$$

Since $\eta \geq \frac{T-2}{2}$, then we get that

$$
\Delta u(t) \leq y(\eta)(1-\alpha(T-2)) t(t-T) \leq 0
$$

So, for $\forall t \in[0, T-1]_{\mathbb{Z}}, \Delta u(t) \leq 0$, which implies that $u(t)$ is decreasing. Since $u(T)=0$, for $\forall t \in[0, T]_{\mathbb{Z}}$, we have $u(t) \geq 0$ and $u \in K_{0}$. For $\forall t \in[1, \eta+1]_{\mathbb{Z}}, \Delta^{2} u(t-1) \leq 0$, we get that $u(t)$ is concave on $[0, \eta+2]_{\mathbb{Z}}$.

Lemma 2.4 Let $\left(H_{0}\right)$ hold. If $y \in K_{0}$, then the solution $u(t)$ of $(2.1)$ satisfies

$$
\min _{t \in[T-\theta, \theta]_{\mathbb{Z}}} u(t)=u(\theta) \geq \frac{\eta+2-\theta}{\eta+2}\|u\|=\theta^{*}\|u\|,
$$

where $\theta^{*}=\frac{\eta+2-\theta}{\eta+2}, \theta \in\left[\left\lfloor\frac{T}{2}\right\rfloor+1, \eta+2\right]_{\mathbb{Z}}$. 
Proof From Lemma 2.3, $u(t)$ is concave on $[0, \eta+2]_{\mathbb{Z}}$. So, $u(t)$ satisfies

$$
\frac{u(t)-u(0)}{t} \geq \frac{u(\eta+2)-u(0)}{\eta+2}, \quad t \in[0, \eta+2]_{\mathbb{Z}} .
$$

Meanwhile, from Lemma 2.3, $u(t)$ is non-increasing on $[0, T]_{\mathbb{Z}}$, which implies that $u(0)=$ $\|u\|$. Therefore,

$$
\begin{aligned}
& u(t) \geq \frac{\eta+2-t}{\eta+2} u(0)=\frac{\eta+2-t}{\eta+2}\|u\|, \\
& \min _{t \in[T-\theta, \theta]_{\mathbb{Z}}} u(t)=u(\theta) \geq \frac{\eta+2-\theta}{\eta+2}\|u\|=\theta^{*}\|u\| .
\end{aligned}
$$

\section{Main results}

$\left(H_{1}\right) f:[1, T-2]_{\mathbb{Z}} \times[0, \infty) \rightarrow[0, \infty)$ is continuous. For $u \in[0, \infty), f(t, u)$ is a decreasing function with respect to $t$, and for $t \in[1, T-2]_{\mathbb{Z}}, f(t, u)$ is an increasing function with respect to $u$.

$\left(H_{2}\right) a:[1, T-2]_{\mathbb{Z}} \rightarrow[0, \infty)$ is a decreasing function.

Let

$$
K=\left\{u \in K_{0} \mid \min _{t \in[T-\theta, \theta]_{\mathbb{Z}}} u(t) \geq \theta^{*}\|u\|\right\} .
$$

Then $K$ is a cone in $E$. Define the operator $S: K \rightarrow E$ as

$$
S u(t)=\sum_{s=1}^{T-2} G(t, s) a(s) f(s, u(s)) .
$$

Lemma 3.1 $S: K \rightarrow K$ is completely continuous.

Proof It is obvious that $S: K \rightarrow E$ is completely continuous since the Banach space $E$ is finite dimensional. Now, let us prove that $S: K \rightarrow K$, that is to say, for any $u \in K$, Su $\in$ K.

Let $u \in K$. Then $u \in K_{0}$, which implies that $\Delta u(t) \leq 0$ and $u$ is decreasing on $t$. Therefore, by $\left(H_{1}\right), f(t, u(t))$ is a decreasing function of $t$. Let $y(t):=a(t) f(t, u(t))$. Then, from $\left(H_{1}\right)$ and $\left(H_{2}\right)$, we obtain that $y(t) \geq 0$ and $y$ is also a decreasing function of $t$. Thus, $y \in K_{0}$. Furthermore, by (3.1), we know that

$$
\Delta^{3}(S u)(t-1)=y(t), \quad t \in[1, T-2]_{\mathbb{Z}},
$$

and

$$
\Delta(S u)(0)=(S u)(T)=0, \quad \Delta^{2}(S u)(\eta)-\alpha \Delta(S u)(T-1)=0 .
$$

Therefore, $S u$ satisfies problem (2.1). Now, similar to the proof of Lemma 2.3, and using the fact $y \in K_{0}$, we obtain that $S u \in K_{0}$ and $S u$ is concave on $[0, \eta]_{\mathbb{Z}}$. Furthermore, by 
Lemma 2.4 and the fact $S u \in K_{0}$, we know that

$$
\min _{t \in[T-\theta, \theta] \mathbb{Z}}(S u)(t) \geq \theta^{*}\|S u\|
$$

Therefore, $S u \in K$ and $S: K \rightarrow K$ is completely continuous.

From (3.1) and Lemma 3.1, we know that if $u$ is a fixed point of $S$ in $K$, then $u$ is a positive solution of (1.1). In the rest of this paper, we try to prove $S$ has at least one or two fixed point(s) in $K$ by using Theorem 1.1 and Theorem 1.2.

Let

$$
A=\sum_{s=1}^{T-2} \frac{T(T-1)(1+\alpha \eta)}{1-\alpha(T-1)} a(s), \quad B=\sum_{s=T-\theta}^{\theta} \frac{\theta(T-\theta)[2-\alpha(\theta-1)]}{2-2 \alpha(T-1)} a(s) .
$$

Theorem 3.1 Suppose that $\left(H_{0}\right),\left(H_{1}\right)$, and $\left(H_{2}\right)$ hold. If there exist two constants $r$ and $R$ $(r \neq R)$ such that

$\left(\mathrm{A}_{1}\right) f(t, u) \leq \frac{r}{A},(t, u) \in[1, T-2]_{\mathbb{Z}} \times[0, r]$

$\left(\mathrm{A}_{2}\right) f(t, u) \geq \frac{R}{B},(t, u) \in[1, T-2]_{\mathbb{Z}} \times\left[\theta^{*} R, R\right]$,

then problem (1.1) has at least one positive solution $u \in K$ with $\min \{r, R\} \leq\|u\| \leq$ $\max \{r, R\}$.

Proof Without loss of generality, suppose that $r<R$, the other case could be treated similarly. Let $\Omega_{1}=\{u \in E:\|u\|<r\}$. From Lemma 2.2, $G(t, s) \leq 0$ for $s \in[\eta+1, T-2]_{\mathbb{Z}}$; $G(t, s) \geq 0$ for $s \in[1, \eta]_{\mathbb{Z}}$. Since $\left(\mathrm{A}_{1}\right)$, we get, for $\forall u \in K \cap \partial \Omega_{1}$,

$$
\begin{aligned}
\|S u\| & =\max _{t \in[0, T]_{\mathbb{Z}}}\left|\sum_{s=1}^{T-2} G(t, s) a(s) f(s, u(s))\right| \\
& \leq \max _{t \in[0, T]_{\mathbb{Z}}} \sum_{s=1}^{T-2}|G(t, s)| a(s) f(s, u(s)) \\
& \leq \sum_{s=1}^{T-2} \frac{T(T-1)(1+\alpha \eta)}{1-\alpha(T-1)} a(s) f(s, u(s)) \\
& \leq \sum_{s=1}^{T-2} \frac{T(T-1)(1+\alpha \eta)}{1-\alpha(T-1)} a(s) \frac{r}{A} \\
& =r .
\end{aligned}
$$

So, for $u \in K \cap \partial \Omega_{1}$,

$$
\|S u\| \leq\|u\| .
$$

Let $\Omega_{2}=\{u \in E:\|u\|<R\}$. Then, for $u \in K \cap \partial \Omega_{2}$,

$$
S u(T-\theta)=\sum_{s=1}^{T-2} G(T-\theta, s) a(s) f(s, u(s)) \geq \sum_{s=\theta}^{T-\theta} G(T-\theta, s) a(s) f(s, u(s)) .
$$


In fact, by Lemma 2.2,

$$
\begin{aligned}
& \sum_{s=1}^{T-\theta-1} G(T-\theta, s) a(s) f(s, u(s))+\sum_{s=\theta+1}^{T-2} G(T-\theta, s) a(s) f(s, u(s)) \\
& \geq \sum_{s=1}^{T-\theta-1} G(T-\theta, s) a(s) f(s, u(s))+\sum_{s=\eta+1}^{T-2} G(T-\theta, s) a(s) f(s, u(s)) \\
& \geq a(\eta) f(\eta, u(\eta))\left[\sum_{s=1}^{T-\theta-1} G(T-\theta, s)+\sum_{s=\eta+1}^{T-2} G(T-\theta, s)\right] .
\end{aligned}
$$

Furthermore,

$$
\begin{array}{rl}
\sum_{s=1}^{T-\theta-1} & G(T-\theta, s)+\sum_{s=\eta+1}^{T-2} G(T-\theta, s) \\
= & -\sum_{s=1}^{T-\theta-1} \frac{\alpha T(T-1)(T-s-1)}{2-2 \alpha(T-1)}-\sum_{s=1}^{T-\theta-1} \frac{(T-s)(T-s-1)}{2} \\
& -\sum_{s=\eta+1}^{T-2} \frac{\alpha T(T-1)(T-s-1)}{2-2 \alpha(T-1)}-\sum_{s=\eta+1}^{T-2} \frac{(T-s)(T-s-1)}{2}+\sum_{s=1}^{T-\theta-1} \frac{T(T-1)}{2-2 \alpha(T-1)} \\
& +\sum_{s=1}^{T-\theta-1} \frac{\alpha(T-s-1)(T-\theta)(T-\theta-1)}{2-2 \alpha(T-1)}+\sum_{s=1}^{T-\theta-1} \frac{(T-\theta-s)(T-\theta-s-1)}{2-2 \alpha(T-1)} \\
& -\sum_{s=1}^{T-\theta-1} \frac{(T-\theta)(T-\theta-1)}{2-2 \alpha(T-1)} .
\end{array}
$$

Let

$$
\begin{aligned}
I_{1}:= & -\sum_{s=1}^{T-\theta-1} \frac{\alpha T(T-1)(T-s-1)}{2-2 \alpha(T-1)}-\sum_{s=1}^{T-\theta-1} \frac{(T-s)(T-s-1)}{2} \\
& -\sum_{s=\eta+1}^{T-2} \frac{\alpha T(T-1)(T-s-1)}{2-2 \alpha(T-1)}-\sum_{s=\eta+1}^{T-2} \frac{(T-s)(T-s-1)}{2} \\
& +\sum_{s=1}^{T-\theta-1} \frac{T(T-1)}{2-2 \alpha(T-1)}
\end{aligned}
$$

and

$$
\begin{aligned}
I_{2}:= & \sum_{s=1}^{T-\theta-1} \frac{\alpha(T-s-1)(T-\theta)(T-\theta-1)}{2-2 \alpha(T-1)}+\sum_{s=1}^{T-\theta-1} \frac{(T-\theta-s)(T-\theta-s-1)}{2-2 \alpha(T-1)} \\
& -\sum_{s=1}^{T-\theta-1} \frac{(T-\theta)(T-\theta-1)}{2-2 \alpha(T-1)} .
\end{aligned}
$$


Then

$$
\begin{aligned}
I_{1} \geq & -\sum_{s=1}^{T-\theta-1}(T-s-1) \frac{(T-1)(1+\alpha)}{2-2 \alpha(T-1)}-\sum_{s=\theta}^{T-2}(T-s-1) \frac{(T-1)(1+\alpha)}{2-2 \alpha(T-1)} \\
& +\sum_{s=1}^{T-\theta-1} \frac{T(T-1)}{2-2 \alpha(T-1)} \\
= & -\frac{(T-1)(1+\alpha)(T-1-\theta)(T-2+\theta)}{4-4 \alpha(T-1)}-\frac{(T-1)(1+\alpha)(T-1-\theta)(T-\theta)}{4-4 \alpha(T-1)} \\
& +\frac{T(T-1)(T-\theta-1)}{2-2 \alpha(T-1)} \\
= & \frac{(T-1)(T-\theta-1)}{2-2 \alpha(T-1)}(T-(1+\alpha)(T-1)) \\
\geq & \frac{(T-1)(T-\theta-1)}{2-2 \alpha(T-1)}\left(T-\frac{T}{T-1}(T-1)\right) \\
= & 0
\end{aligned}
$$

and

$$
\begin{aligned}
I_{2} & =\frac{(T-\theta)(T-\theta-1)(T-\theta-1)}{2-2 \alpha(T-1)}(\alpha(T-2+\theta)-1)+\sum_{s=1}^{T-\theta-1} \frac{(T-\theta-s)(T-\theta-s-1)}{2-2 \alpha(T-1)} \\
& =\frac{(T-\theta)(T-\theta-1)(T-\theta-1)}{2-2 \alpha(T-1)}(\alpha(T-2+\theta)-1)+\frac{(T-\theta)(T-\theta-1)(T-\theta-2)}{3} \\
& =\frac{(T-\theta)(T-\theta-1)(T-\theta-2)}{6-6 \alpha(T-1)}[\alpha(T-8)+3(\theta-1)] \\
& \geq 0 .
\end{aligned}
$$

Therefore, (3.5) holds. This implies that

$$
\begin{aligned}
\operatorname{Su}(T-\theta) \geq & \sum_{s=T-\theta}^{\theta} G(T-\theta, s) a(s) f(s, u(s)) \\
= & \sum_{s=T-\theta}^{\theta}\left\{\frac{1-\alpha(T-s-s)[T(T-1)-(T-\theta)(T-\theta-1)]}{2-2 \alpha(T-1)}\right. \\
& \left.-\frac{(T-s)(T-s-1)}{2}+\frac{(T-\theta-s)(T-\theta-s-1)}{2}\right\} a(s) f(s, u(s)) \\
\geq & \sum_{s=T-\theta}^{\theta} \frac{\theta(T-\theta)[2-\alpha(\theta-1)]}{2-2 \alpha(T-1)} a(s) f(s, u(s)) \\
\geq & \sum_{s=T-\theta}^{\theta} \frac{\theta(T-\theta)[2-\alpha(\theta-1)]}{2-2 \alpha(T-1)} a(s) \frac{R}{B} \\
\geq & R .
\end{aligned}
$$

So, for $\forall u \in K \cap \partial \Omega_{2}$, 
Then, by Theorem 1.1, $S$ has at least one fixed point $u \in K$ and $u$ will be a positive solution of problem (1.1).

Theorem 3.2 Suppose that $\left(H_{0}\right),\left(H_{1}\right)$, and $\left(H_{2}\right)$ hold. If one of the following conditions holds:

(B) B $\left._{1}\right):=\lim _{u \rightarrow 0^{+}} \max _{t \in[1, T-2]_{\mathbb{Z}}} \frac{f(t, u)}{u}=0, \quad f_{\infty}:=\lim _{u \rightarrow \infty} \max _{t \in[1, T-2]_{\mathbb{Z}}} \frac{f(t, u)}{u}=\infty$,

or

$\left(B_{2}\right) \quad f_{0}:=\lim _{u \rightarrow 0^{+}} \max _{t \in[1, T-2]_{\mathbb{Z}}} \frac{f(t, u)}{u}=\infty, \quad f^{\infty}:=\lim _{u \rightarrow \infty} \max _{t \in[1, T-2]_{\mathbb{Z}}} \frac{f(t, u)}{u}=0$,

then (1.1) has at least one positive solution.

Proof Firstly, we prove the case that $\left(B_{1}\right)$ holds. Since $f^{0}=0$, there exists a constant $R_{1}>0$ such that

$$
\frac{f(t, u)}{u} \leq \frac{R_{1}}{A}, \quad(t, u) \in[1, T-2]_{\mathbb{Z}} \times\left[0, R_{1}\right] .
$$

Since $f_{\infty}=\infty$, there exists a constant $R_{2}>R_{1}$ such that

$$
f(t, u) \geq \frac{u}{\theta^{*} B} \geq \frac{\theta^{*} R_{2}}{\theta^{*} B}=\frac{R_{2}}{B}, \quad(t, u) \in[1, T-2]_{\mathbb{Z}} \times\left[\theta^{*} R_{2}, R_{2}\right] .
$$

From Theorem 3.1, problem (1.1) has at least one positive solution $u \in K$.

Secondly, suppose that $\left(B_{2}\right)$ holds. Since $f_{0}=\infty$, there exists a constant $r_{1}>0$ such that

$$
f(t, u) \geq \frac{u}{\theta^{*} B}, \quad(t, u) \in[1, T-2]_{\mathbb{Z}} \times\left[0, r_{1}\right] .
$$

Let $\Omega_{1}=\left\{u \in E:\|u\|<r_{1}\right\}$. If $u \in K \cap \partial \Omega_{1}$, we have

$$
\min _{s \in[T-\theta, \theta]_{\mathbb{Z}}} u(s) \geq \theta^{*}\|u\|=\theta^{*} r_{1} .
$$

Therefore, similar to the proof of (3.6), we have

$$
\|S u\| \geq\|u\| \quad \text { for } u \in K \cap \partial \Omega_{1} .
$$

On the other hand, since $f^{\infty}=0$, there exists a constant $r_{2}>0$ such that

$$
f(t, u) \leq \frac{u}{A}, \quad(t, u) \in[1, T-2]_{\mathbb{Z}} \times\left[r_{2}, \infty\right)
$$

If $f$ is bounded, then there exists a constant $N>0$ such that $f \leq N$. So, we choose $R^{\prime}=\max \left\{2 r_{1}, N A\right\}$. If $f$ is unbounded, then let $R^{\prime}>\max \left\{2 r_{1}, r_{2}\right\}$ such that $f(t, u) \leq f\left(t, R_{2}\right)$. Let $\Omega_{2}=\left\{u \in K:\|u\|<R^{\prime}\right\}$ for $\forall(t, u) \in[1, T-2]_{\mathbb{Z}} \times\left[0, R_{2}\right]$. Similar to the proof of Theorem 3.1, we get, for $\forall u \in k \cap \partial \Omega_{2}$,

$$
\|S u\| \leq\|u\| .
$$


Thus, by Theorem 1.1, $S$ has at least one fixed point $u \in K \cap \overline{\Omega_{2}} \backslash \Omega_{1}$, which is a positive solution of problem (1.1).

Theorem 3.3 Assume that $\left(H_{0}\right),\left(H_{1}\right)$, and $\left(H_{2}\right)$ hold. If

$\left(C_{1}\right) f_{0}:=\lim _{u \rightarrow 0^{+}} \min _{t \in[1, T-2]_{\mathbb{Z}}}=\frac{f(t, u)}{u}=+\infty, f_{\infty}:=\lim _{u \rightarrow \infty} \min _{t \in[1, T-2]_{\mathbb{Z}}}=\frac{f(t, u)}{u}=+\infty$, and

$\left(C_{2}\right)$ There exists a constant $p>0$ such that $f(t, u)<\gamma p$ for $0 \leq u \leq p$ and $t \in[1, T-2]_{\mathbb{Z}}$, where

$$
\gamma=\left(\frac{T(T-1)(1+\alpha \eta)}{1-\alpha(T-1)} \sum_{s=1}^{T-2} a(s)\right)^{-1}
$$

then problem (1.1) has at least two positive solutions $u_{1}$ and $u_{2}$ with $0 \leq\left\|u_{1}\right\| \leq p \leq\left\|u_{2}\right\|$.

Proof Choose $M>0$ such that

$$
M \theta^{*} \frac{\theta(T-\theta)[2-\alpha(\theta-1)]}{2-2 \alpha(T-1)} \sum_{s=T-\theta}^{\theta} a(s) \geq 1
$$

Since $f_{0}=+\infty$, there exists a constant $r$ with $0<r<p$ such that $f(t, u) \geq M u$ for $0 \leq u \leq r$. Then, for $\forall u \in \partial K_{r}$, we have

$$
\begin{aligned}
\operatorname{Su}(T-\theta) & =\sum_{s=1}^{T-2} G(T-\theta, s) a(s) f(s, u(s)) \\
& \geq \sum_{s=T-\theta}^{\theta} G(T-\theta, s) a(s) f(s, u(s)) \\
& \geq M \theta^{*} \sum_{s=T-\theta}^{\theta} G(T-\theta, s) a(s)\|u\| \\
& \geq M \theta^{*} \frac{\theta(T-\theta)[2-\alpha(\theta-1)]}{2-2 \alpha(T-1)} \sum_{s=T-\theta}^{\theta} a(s)\|u\| \\
& \geq\|u\| .
\end{aligned}
$$

From Theorem 1.2, we get

$$
i\left(S, K_{r}, K\right)=0 .
$$

Since $f_{\infty}=+\infty$, there exists a constant $R_{1}>0$ such that $f(t, u) \geq M u$ for $\forall u \geq R_{1}$. Choose $R>\max \left\{p, \frac{R_{1}}{\theta^{*}}\right\}$, then for $\forall u \in \partial K_{R}, \min _{t \in[T-\theta, \theta]_{\mathbb{Z}}} u(t) \geq \theta^{*}\|u\|>R_{1}$. Similar to the above proof, we have

$$
\|S u\| \geq\|u\| \quad \text { for } u \in \partial K_{R} .
$$

Therefore,

$$
i\left(S, K_{R}, K\right)=0 .
$$


From $\left(C_{2}\right)$, for $\forall u \in \partial K_{p}$,

$$
\begin{aligned}
\|S u\| & =\max _{t \in[0, T]_{\mathbb{Z}}}\left|\sum_{s=1}^{T-2} G(t, s) a(s) f(s, u(s))\right| \\
& \leq \max _{t \in[0, T]_{\mathbb{Z}}} \sum_{s=1}^{T-2}|G(t, s)| a(s) f(s, u(s)) \\
& \leq \sum_{s=1}^{T-2} \frac{T(T-1)(1+\alpha \eta)}{1-\alpha(T-1)} a(s) f(s, u(s)) \\
& =\|u\| .
\end{aligned}
$$

Therefore, for $\forall u \in \partial K_{p},\|T u\| \leq\|u\|$. By Theorem 1.2,

$$
i\left(S, K_{p}, K\right)=1 \text {. }
$$

Thus,

$$
i\left(S, K_{R} \backslash \stackrel{\circ}{K}_{p}, K\right)=-1, \quad i\left(S, K_{p} \backslash \stackrel{\circ}{K}_{r}, K\right)=1 .
$$

So, $S$ has a fixed point $u_{1}$ in $K_{p} \backslash \stackrel{\circ}{K}_{r}$ and another fixed point $u_{2}$ in $K_{R} \backslash \stackrel{\circ}{K}_{p}$. So, problem (1.1) has at least two positive solutions $u_{1}$ and $u_{2}$ with $0 \leq\left\|u_{1}\right\| \leq p \leq\left\|u_{2}\right\|$.

Theorem 3.4 Assume that $\left(H_{0}\right),\left(H_{1}\right)$, and $\left(H_{2}\right)$ hold. If

$\left(D_{1}\right) f^{0}:=\lim _{u \rightarrow 0^{+}} \max _{t \in[1, T-2]_{\mathbb{Z}}} \frac{f(t, u)}{u}=0, f^{\infty}:=\lim _{u \rightarrow \infty} \max _{t \in[1, T-2]_{\mathbb{Z}}} \frac{f(t, u)}{u}=0$, and

$\left(D_{2}\right)$ there exists a constant $p>0$ such that $f(t, u)>\beta p$ for $\theta^{*} p \leq u \leq p$ and $t \in[1, T-2]_{\mathbb{Z}}$, where

$$
\beta=\left(\theta^{*} \frac{\theta(T-\theta)[2-\alpha(\theta-1)]}{2-2 \alpha(T-1)} \sum_{s=T-\theta}^{\theta} a(s)\right)^{-1}
$$

then (1.1) has at least two positive solutions $u_{1}$ and $u_{2}$ with $0 \leq\left\|u_{1}\right\| \leq p \leq\left\|u_{2}\right\|$.

Proof From $\left(D_{1}\right)$, for $\forall \epsilon>0$, there exists $M_{1}>0$, if $u>0, t \in[0, T]_{\mathbb{Z}}$, we have $f(t, u) \leq$ $M_{1}+\epsilon u$. Then, for $\forall u \in K$,

$$
\begin{aligned}
\|S u\| & =\max _{t \in[0, T]_{\mathbb{Z}}}\left|\sum_{s=1}^{T-2} G(t, s) a(s) f(s, u(s))\right| \\
& \leq \frac{T(T-1)(1+\alpha \eta)}{1-\alpha(T-1)} \sum_{s=1}^{T-2} a(s)\left(M_{1}+\epsilon u\right) .
\end{aligned}
$$

Choose $\epsilon>0$ sufficiently small and $R>p$ sufficiently large, then for $\forall u \in \partial K_{R},\|S u\| \leq\|u\|$, from Theorem 1.2, we have

$$
i\left(S, K_{R}, K\right)=1 .
$$


In a similar way, if $0<r<p$,

$$
i\left(S, K_{R}, K\right)=1
$$

From $\left(D_{2}\right)$, for $\forall u \in \partial K_{p}$,

$$
\begin{aligned}
\operatorname{Su}(T-\theta) & =\sum_{s=1}^{T-2} G(T-\theta, s) a(s) f(s, u(s)) \\
& \geq \sum_{s=T-\theta}^{\theta} G(T-\theta, s) a(s) f(s, u(s)) \\
& >\beta \theta^{*} p \sum_{s=T-\theta}^{\theta} G(T-\theta, s) a(s) \\
& \geq \beta \theta^{*} p \frac{\theta(T-\theta)[2-\alpha(\theta-1)]}{2-2 \alpha(T-1)} \sum_{s=T-\theta}^{\theta} a(s) \\
& =p .
\end{aligned}
$$

Then, for $\forall u \in \partial K_{p},\|S u\| \geq\|u\|$. From Theorem 1.2, we have

$$
i\left(S, K_{p}, K\right)=0
$$

Then, problem (1.1) has at least two solutions $u_{1}$ and $u_{2}$ with $0 \leq\left\|u_{1}\right\| \leq p \leq\left\|u_{2}\right\|$.

\section{Example}

Example 4.1 Consider the discrete three-point boundary problem

$$
\left\{\begin{array}{l}
\Delta^{3} u(t-1)-a(t) f(t, u(t))=0, \quad t \in[1,7]_{\mathbb{Z}} \\
\Delta u(0)=u(9)=0, \quad \Delta^{2} u(7)-\frac{1}{9} \Delta u(8)=0
\end{array}\right.
$$

where $a(t)=\frac{9-t}{10}$, and

$$
f(t, u)= \begin{cases}15-t+\frac{u}{1000}, & (t, u) \in[1,7]_{\mathbb{Z}} \times[0,1000] \\ \sqrt[3]{u}+6-t, & (t, u) \in[1,7]_{\mathbb{Z}} \times[1000, \infty]\end{cases}
$$

Since $T=9$ and $\alpha=\frac{1}{9}, \eta \in[4,7]_{\mathbb{Z}}$. Without loss of generality, let $\eta=4$. Then, by the direct calculation, we get $\theta \in[5,6]_{\mathbb{Z}}$. Choose $\theta=5$, then $\theta^{*}=\frac{\eta+2-\theta}{\eta+2}=\frac{1}{6}$. So,

$$
\begin{aligned}
& A=\sum_{s=1}^{T-2} \frac{T(T-1)(1+\alpha \eta)}{1-\alpha(T-1)} a(s)=936 \sum_{s=1}^{7} \frac{9-s}{10}=3276 \\
& B=\sum_{s=T-\theta}^{\theta} \frac{\theta(T-\theta)[2-\alpha(\theta-1)]}{2-2 \alpha(T-1)} a(s)=140 \sum_{s=4}^{5} \frac{9-s}{10}=136 .
\end{aligned}
$$

If we choose $R=330, r=1,000,000$, from Theorem 3.1, problem (4.1) has at least one positive solution. 
Example 4.2 In this example, we continue to consider problem (4.1) with

$$
f(t, u)= \begin{cases}\frac{u^{2}(10-t)}{10}, & (t, u) \in[1,7]_{\mathbb{Z}} \times[0,1], \\ \frac{\sqrt[3]{u}+9-t}{10}, & (t, u) \in[1,7]_{\mathbb{Z}} \times[1,3], \\ \frac{\sqrt[3]{u}+1890-t}{1000}, & (t, u) \in[1,7]_{\mathbb{Z}} \times[3, \infty) .\end{cases}
$$

Continue to take $\alpha=\frac{1}{9}, \eta=4, \theta=5$, and $\theta^{*}=\frac{1}{6}$. Then

$$
\beta=\left(\theta^{*} \frac{\theta(T-\theta)[2-\alpha(\theta-1)]}{2-2 \alpha(T-1)} \sum_{s=T-\theta}^{\theta} a(s)\right)^{-1}=\frac{3}{68} .
$$

Furthermore, if we choose $p=1$, then for $\theta^{*} p \leq u \leq p, f(t, u) \geq \beta p=\frac{3}{68}$. From Theorem 3.4, problem (4.1) has at least two positive solutions $u_{1}$ and $u_{2}$ with $0 \leq\left\|u_{1}\right\| \leq p \leq$ $\left\|u_{2}\right\|$.

\section{Acknowledgements}

The authors express their gratitude to the referee for reading the paper carefully and making several corrections and remarks.

\section{Funding}

This work was supported by the National Natural Science Foundation of China (No. 71561024). The key projects of Natural Science Foundation of Gansu province of China (No. 18JR3RA084).

\section{Availability of data and materials}

The datasets used and analysed during the current study are available from the corresponding author on reasonable request.

\section{Competing interests}

The authors declare that they do not have any competing interests in this manuscript.

\section{Consent for publication}

The authors confirm that the work described has not been published before (except in the form of an abstract or as part of a published lecture, review, or thesis), that its publication has been approved by all co-authors.

\section{Authors' contributions}

All authors contributed equally to this manuscript. All authors read and approved the final manuscript.

\section{Publisher's Note}

Springer Nature remains neutral with regard to jurisdictional claims in published maps and institutional affiliations.

Received: 30 January 2019 Accepted: 17 May 2019 Published online: 28 May 2019

\section{References}

1. Il'in, V.A., Mosiseev, E.I.: Nonlocal boundary value problem of the first kind for a Sturm-Liouville operator in its differential and finite difference aspects. Electron. J. Differ. Equ. 23, 803 (1987)

2. Gupta, C.P.: Solvability of a three points nonlinear boundary value problem for a second order differential equation. J. Math. Anal. Appl. 168, 540-551 (1992)

3. Feng, W., Webb, J.R.L.: Solvability of three-point boundary value problem at resonance. Nonlinear Anal. 30, 322-323 (1997)

4. Gupta, C.P., Trofimchuk, S.I.: Solvability of a multipoint boundary value problem and related a priori estimates. Can. Appl. Math. Q. 6, 45-60 (1995)

5. Han, B., Yang, Y: An integro-PDE model with variable motility. Nonlinear Anal., Real World Appl. 45, 186-199 (2019)

6. Liu, L., Liu, B., Wu, Y.: Nontrivial solutions for higher-order $m$-point boundary value problem with a sign-changing nonlinear term. Appl. Math. Comput. 217, 3792-3800 (2010)

7. Ma, R.: Positive solutions for a nonlinear three-point boundary value problem. Electron. J. Differ. Equ. 34, 1 (1999)

8. Liu, B., Liu, L., Wu, Y.: Multiple solutions of singular three-point boundary value problems on $[0, \infty)$. Nonlinear Anal. 70, 3348-3357 (2009)

9. Cao, D., Ma, R.: Positive solutions to a second order multipoint boundary value problem. Electron. J. Differ. Equ. 2000, 65 (2000)

10. Eloe, P., Henderson, J.: Positive solutions and nonlinear multipoint conjugate eigenvalue problems. Electron. J. Differ. Equ. 1997, 3 (1997) 
11. Guo, Y., Shan, W., Ge, W.: Positive solutions for second-order m-point boundary value. J. Comput. Appl. Math. 151, 415-424 (2003)

12. Ji, J., Yang, B.: Positive solutions of discrete third-order three-point right focal boundary value problems. J. Differ. Equ. Appl. 15(2), 185-195 (2009)

13. Liu, L., Hao, X., Wu, Y.: Unbounded solutions of second-order multipoint boundary value problem on the half-line. Bound. Value Probl. 2010, Article ID 236560 (2010)

14. Hao, X., Liu, L., Wu, Y.: On positive solutions of an $m$-point nonhomogeneous singular boundary value problem. Nonlinear Anal. 73, 2532-2540 (2010)

15. Ma, R.: Existence of positive solutions for superlinear semipositone $m$-point boundary value problems. Proc. Edinb. Math. Soc. 46, 279-292 (2003)

16. Ma, R.: Positive solutions of a nonlinear m-point boundary value problem. Comput. Math. Appl. 42, 755-765 (2001)

17. Shi, Q., Wang, S.: Klein-Gordon-Zakharov system in energy space: blowup profile and subsonic limit. Math. Methods Appl. Sci. (2019). https://doi.org/10.1002/mma.5579

18. Song, Q., Bai, Z:: Positive solutions of fractional differential equations involving the Riemann-Stieltjes integral boundary condition. Adv. Differ. Equ. 2018, 183 (2018)

19. Bai, Z., Zhang, Y.: Solvability of fractional three-point boundary value problems with nonlinear growth. Appl. Math. Comput. 218(5), 1719-1725 (2011)

20. Agarwal, R.P., Perera, K., O'Regan, D.: Multiple positive solutions of singular discrete $p$-Laplacian problems via variational methods. Adv. Differ. Equ. 2005, 2 (2005)

21. Gao, C., Li, X., Zhang, F.: Eigenvalues of discrete Sturm-Liouville problems with nonlinear eigenparameter dependent boundary conditions. Quaest. Math. 41,773-797 (2018)

22. Gao, C., Ma, R., Zhang, F.: Spectrum of discrete left definite Sturm-Liouville problems with eigenparameter-dependent boundary conditions. Linear Multilinear Algebra 65, 1905-1923 (2017)

23. Karaca, I.Y.: Discrete third-order three-point boundary value problem. J. Comput. Appl. Math. 205, 458-468 (2007)

24. Agarwal, R.P., Henderson, J.: Positive solutions and nonlinear eigenvalue problems for third-order difference equations. Comput. Math. Appl. 36, 347-355 (1998)

25. Wang, J., Gao, C.: Positive solutions of discrete third-order boundary value problems with sign-changing Green's function. Adv. Differ. Equ. 2015, 56 (2015)

26. Gao, C., Geng, T.: Positive solutions of a discrete nonlinear third-order three-point eigenvalue problem with sign-changing Green's function. Adv. Differ. Equ. 2016, 110 (2016)

27. Gao, C., Zhang, F., Ma, R.: Existence of positive solutions of second-order periodic boundary value problems with sign-changing Green's function. Acta Math. Appl. Sin. Engl. Ser. 33, 263-268 (2017)

28. Gao, L., Sun, J.: Positive solutions of a third-order three-point BVP with sign-changing Green's function. Math. Probl. Eng. 2014, Article ID 406815 (2014)

29. Niu, B., Sun, J., Ren, Q.: Two positive solutions of third-order BVP with integral boundary condition and sign-changing Green's function. J. Funct. Spaces 2015, Article ID 491423 (2015)

30. Guo, D., Lakshmikantham, V.: Nonlinear Problems in Abstract Cones. Academic Press, San Diego (1988)

\section{Submit your manuscript to a SpringerOpen ${ }^{\circ}$ journal and benefit from:}

- Convenient online submission

- Rigorous peer review

- Open access: articles freely available online

- High visibility within the field

- Retaining the copyright to your article

Submit your next manuscript at $\gg$ springeropen.com 